\title{
Narrativa
}

\section{Raccontare il sud. La narrativa in terra di Bari}

\section{Giovanna Zaccaro}

\section{OpenEdition}

\section{Journals}

\section{Edizione digitale}

URL: https://journals.openedition.org/narrativa/712

DOI: $10.4000 /$ narrativa. 712

ISSN: 2804-1224

\section{Editore}

Presses universitaires de Paris Nanterre

\section{Edizione cartacea}

Data di pubblicazione: 1 décembre 2017

Paginazione: 149-160

ISBN: 978-2-84016-289-6

ISSN: $1166-3243$

\section{Notizia bibliografica digitale}

Giovanna Zaccaro, «Raccontare il sud. La narrativa in terra di Bari», Narrativa [Online], 39 | 2017, online dal 01 décembre 2021, consultato il 14 janvier 2022. URL: http://journals.openedition.org/narrativa/ 712 ; DOI: https://doi.org/10.4000/narrativa. 712

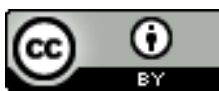

Narrativa est mise à disposition selon les termes de la Licence Creative Commons Attribution 4.0 International. 


\section{Raccontare il sud. La narrativa in terra di Bari}

$\mathrm{P}$ er raccontare il Sud di oggi, una realtà sempre più complessa e sfuggente, sempre più plurale, per provare a costruire un atlante letterario della Puglia inteso a dare conto dei mutamenti, della progettualità in atto, delle pratiche di scrittura che si stanno sperimentando e che provocano le trasformazioni dei valori letterari ${ }^{1}$, per restituire al Sud "l'antica dignità di soggetto del pensiero", è necessario mappare le voci che ci aiutano a scoprire quanto ci sia di unificante, di specifico e di nuovo nel Mezzogiorno, e in particolare in Puglia, in terra di Bari. Una terra del Mediterraneo, il mare fra le terre, un mare pluriverso, che mette al centro il confine, un confine che, come afferma Cassano, "non è il luogo dove il mondo finisce, ma quello dove i diversi si toccano e la partita del rapporto con l'altro diventa difficile e vera"3 e ci obbliga al confronto, riconoscendoci come identità in relazione, e a praticare l'incontro e il dialogo, sperimentando l'ibridazione.

Raccontare il sud a partire dal sud è un compito che abbiamo tentato di assolvere già dal novembre del 2009, con il convegno "Sguardi dai \sui Sud: Meridione, Mediterraneo e Sud globale", tenutosi a Bari e organizzato insieme al collega Luigi Cazzato, in cui si è voluto raccontare, conoscere, criticare, "difendere" il sud nella convinzione che bisogna continuare a indicare strade e prospettive partendo dal sud, nella consapevolezza che il sud ha un pensiero autonomo da avanzare ${ }^{4}$. Abbiamo ritenuto importante raccontare il sud a

1. Cfr. Catalano, Ettore, "Le ragioni di un convegno: la Puglia letteraria tra Regione, Nazione e prospettive mediterranee", in ID. (a cura di), La saggezza della letteratura, Atti del forum letterario "Puglia letteraria, Mediterraneo, Europa", Brindisi, Bari, Edizioni Giuseppe Laterza, 2005, p. 13.

2. Cassano, Franco, Il pensiero meridiano, Bari, Laterza, 2005, p. 5.

3. Ibid, p. 7.

4. Cfr. Cazzato, Luigi (a cura di), Orizzonte Sud, Nardò, Besa, 2011. Da quella esperienza è nato il progetto di ricerca "S $\backslash$ Murare il Mediterraneo" che punta la 
cominciare da Bari per alcune sue specificità culturali, come quella di avere un santo patrono come San Nicola, ovvero un uomo nero venuto dal mare, da una città "levantina" dove i commerci hanno favorito scambi, ibridazioni, incroci. Una città che ha visto nascere una scuola ("les barisiens", dall'appellativo che i parigini diedero al nostro Ricciotto Canudo ${ }^{5}$ ) di scrittori, critici, intellettuali non più abitanti "una provincia dell'impero", ma una "periferia", e proprio per questo aperta all'ascolto e alla sperimentazione.

Proponiamo quindi la ricostruzione, per campioni che riteniamo significativi, di un quadro della narrativa in terra di Bari a partire dagli anni Settanta impegnandoci a seguire un criterio di costruzione non solo critico, ma anche propositivo, nel tentativo di tracciare una linea di interpretazione trasversale all'idea stessa di meridione in letteratura. Insomma, un criterio non di selezione, ma di paradigma, di modello d'interpretazione, volendo implementare un canone "plurale" in cui il pensiero legato alla meridionalità emerga alla luce delle differenze, ma anche delle continuità, delle "costanti"'. Tenendo conto non solo dell'impegno socio-politico rivolto alla condizione del Meridione degli scrittori selezionati, ma anche dell'importanza a loro riconosciuta (l'accoglimento dei loro lavori da case editrici nazionali, l'attenzione critica ricevuta, il successo di vendite, ecc.), le voci che abbiamo incontrato (qui presentate con necessitata selezione) sono cariche di rabbia, disperazione, speranza, ironia, impegno.

A partire dagli anni Ottanta, Raffaele Nigro e Lino Angiuli, redigendo i "Preliminari per un manifesto dell'arte post rurale o dell'occidentalismo imperfetto" ", avviano la loro riflessione sull'occidentalismo imperfetto dichiarando la volontà di mettere la scrittura artistica a servizio di un impegno che promuova incroci ed incontri di uomini e culture, riconoscendo a una regione di frontiera come la Puglia una funzione protagonistica e invitando a una generale mobilitazione intellettuali, artisti, scrittori ("il polline culturale" del nuovo millennio di cui

sua indagine sull'oggi delle migrazioni e prova a mettere a frutto saperi disciplinari che appartengono a vettori diversi perché linguistici, letterari, semiotici, cercando di "smurare" anche muri che sembrano impenetrabili, in una prospettiva interculturale che consente di favorire relazioni, sinergie tra le diversità.

5. Fu Apollinaire ad attribuirglielo quando conobbe Canudo che frequentava i gruppi dell'avanguardia letteraria e artistica a Parigi. Sull'argomento, cfr. Dotoli, Giovanni, Bibliografia critica di Ricciotto Canudo, Fasano, Schena, 1983.

6. Modello di questo percorso anche il volume di CARmosino, Daniela, Uccidiamo la luna a Marechiaro, Roma, Donzelli, 2009.

7. Angiuli, Lino, Nigro, Raffaele, "Preliminari per un manifesto dell'arte postrurale o dell'occidentalismo imperfetto", in Inoltre, n. 1, marzo 1988. 
parla Nigro ${ }^{8}$, per costruire attraverso il ponte della letteratura, come già Calvino sosteneva ${ }^{9}$, una nuova civiltà. Per contribuire così alla costruzione di una nuova identità culturale nella quale Sud ed Europa (il binomio bodiniano: "Il Sud ci fu padre e nostra madre l'Europa"10 si articolano in una sorta di antropologia dei sud del mondo, abbracciando rivoluzioni, sconfitte e scomparse di popoli, culture e parole che, dissepolte e riproblematizzate, contribuiscono a definire i contorni del continente della scrittura creativa aprendosi a una nuova visione pragmatica della cultura, nello scenario di un Mediterraneo letto come spazio conflittuale di culture, economie e fedi diverse, ma in cui si può e si deve costruire il dialogo. Una nuova lettura del sud, o meglio dei sud al plurale, come risorse escluse dalle scene del divenire, riconoscendo intersezioni fra culture. Un'idea del sud che delimita un orizzonte vasto e omogeneo quale il bacino del Mediterraneo, in grado di superare il problema del meridionalismo per abbracciare la questione meridiana ${ }^{11}$. Per dire questo sud serve un'arte che guardi con attenzione alle periferie del mondo, ma a partire dalla consapevolezza di un occidentalismo imperfetto proprio perché conscio dei suoi limiti e dei suoi ritardi. Di qui la ricerca di nuovi linguaggi, di un'arte non ideologica, che si apra ad accogliere le voci, le energie, i bisogni rimossi e negati che chiedono con forza di contare sulla scena del mondo. La scrittura, in questo modo, diventa il laboratorio dove analizzare tale fenomeno: una letteratura che accarezza il fascino dell'altrove e si proietta quale strumento capace di individuare ogni remota parvenza di Itaca $\mathrm{o}$, per essere ancora più precisi, per riconoscere le ardite architetture della "città del sole". Un'arte che consenta anche una ridefinizione dell'identità intellettuale e letteraria, uscendo definitivamente dagli stereotipi, per incontrare se stesso e l'altro. Dallo scontro all'incontro, all'ascolto, all'accoglimento, al riconoscimento delle esigenze di chi chiede. Questo compito, Raffaele Nigro lo ribadisce, spetta agli intellettuali innanzitutto, che devono essere capaci di costruire un inedito sentimento, epico e tragico insieme, che dagli esodi verso l'Europa trae il suo oggettivo supporto. La cultura come principio del riscatto sociale e come motore della storia è, dunque, per Nigro, una costante dell'utopia.

8. Cfr. Nigro, Raffaele, Diario mediterraneo, Bari, Laterza, 2001.

9. Cfr. Calvino, Italo, Lezioni americane. Sei proposte per il nuovo millennio, Milano, Garzanti, 1988.

10. Così il poeta leccese, nell'ultima lirica che chiude Dopo la luna (1956) dal titolo Troppo rapidamente, Caltanisetta-Roma, Sciascia,1956.

11. Cfr. Cassano, Franco, Il pensiero meridiano, cit. 
Si spiega allora come alle origini della produzione narrativa che parte negli anni Settanta (di quella che appunto si colloca in questo alveo) sta Tommaso Fiore con il suo Il cafone all'inferno ${ }^{12}$, in cui l'autore analizza la realtà in movimento delle masse contadine alla luce del suo umanesimo democratico che gli consente di aprirsi verso quell'intelligenza poetica di poesie e di cose, come Calvino gli riconosceva, di trasformare quella che ancora Calvino chiama la "lingua della miseria" nelle parole nuove del suo progetto politico in cui il meridione è storicamente percepito come laboratorio politico ${ }^{13}$.

Gli anni Settanta si aprono con l'ultima produzione di Nino Palumbo di cui ricordiamo i romanzi Il serpente malioso e Domanda marginale ${ }^{14}$ in cui conferma la scelta del realismo critico associandolo allo scandaglio delle zone d'ombra del soggetto piccolo-borghese, un uomo senza qualità stritolato da un sistema burocratico-politico. Il realismo critico approda così nella terra del sogno, della memoria, dell'interiorità.

Sempre negli anni Settanta ha grande rilievo la produzione letteraria di Tommaso Di Ciaula che, col romanzo Tuta Blu ${ }^{15}$, dà voce a quel cafone all'inferno di cui Fiore ci aveva dato il ritratto che, diventato operaio nella zona industriale di Bari (è romanzo autobiografico, la forma è quella del diario, del memoriale), vive con rancore e rimpianto la perdita dal mondo contadino e con rabbia per le condizioni di vita della fabbrica, per la degradazione che subisce come operaio costretto alle logiche del profitto e del rendimento e che racconta, appunto, mescolando il sogno con la rabbia. Il romanzo apriva infatti la strada alla sperimentazione di forme di scrittura antropologica sulla condizione del sud. In seguito Di Ciaula ha continuato a scrivere opere che conservano il tema ribellistico del suo più importante romanzo. Infatti, ribelle è anche il protagonista di Acque sante, acque marce ${ }^{16}$ che, in compagnia di un leone e di un ranocchio, erra nelle terre pugliesi portando acqua santa per purificare le acque marce

12. Fiore, Tommaso, Il cafone all'inferno, Torino, Einaudi, 1955.

13. "Un libro esemplare - scrisse Calvino - come ce ne sarebbe bisogno se ne scrivessero tanti, per ogni regione, per ogni problema. Mi piace poi per l'immagine che dà del Meridione come un paese in movimento, che esprime forze nuove, che può e deve essere diverso: non la solita 'lagna' delle miserie, dunque, ma un piglio ben più risoluto" (ora in FIORE, Vittore (a cura di), Tommaso Fiore e la Puglia, Bari, Palomar, 1996, pp. 497-498).

14. Palumbo, Nino, Il serpente malioso, Roma, Editori Riuniti, 1977; ID. Domanda marginale, Foggia, Bastogi, 1982.

15. Di Ciaula, Tommaso, Tute Blu, Milano, Feltrinelli, 1978.

16. ID., Acque sante, acque marce, Palermo, Sellerio, 1997. 
dall'inquinamento. Sullo sfondo quindi appare una terra mitica, arcaica, magica, la Puglia, protagonista anche dei suoi racconti.

La forte attenzione per la narrativa pugliese da parte della grande editoria, e in particolar modo della Feltrinelli (in seguito sarà dell'Einaudi), è confermata dal fatto che nel giro di quattro anni uscirono per l'editore milanese non solo i due romanzi di Di Ciaula, ma anche Analisi in famiglia ${ }^{17}$ di Maria Marcone e Nero di Puglia ${ }^{18}$ di Antonio Campobassso, quattro libri, tutti autobiografici, che attraverso le storie dei protagonisti rispecchiano la realtà sociale pugliese in profonda trasformazione e rappresentano la complessa e contraddittoria stagione del realismo sociale barese.

Nero è figlio illegittimo di una donna pugliese, Campobasso racconta la sua esperienza in Nero di Puglia, in cui denuncia la violenza di cui è stato vittima. Oltre che un'autobiografia, il romanzo è una "singolare cantata meridionaleafricana" 19 avente come leitmotiv la schiavitù e il dolore. Attraverso sequenze di versi narrativi e lirici, in una forma di poesia/racconto/grido, il libro narra la storia di un personaggio pugliese dalla pelle nera che soffre nella sua "diversità" tutte le pene degli emarginati e dei "diversi" e nello stesso tempo testimonia una disperata voglia di esistere, di resistere, di affermare la propria umanità e desiderio di vita. Un libro "disperato, ateo e ribelle", che al di là del valore testimoniale e anche di un interessante strutturazione prosimetrica, che apre squarci di canto o di invettiva in versi all'interno del tessuto della narrazione, testimonia l'appartenenza alla tesa stagione degli anni Settanta ${ }^{20}$.

L'altro esempio di una scrittura impegnata sul duplice versante della denuncia sociale e dell'introspezione psicologica è quello di Maria Marcone. Nei suoi romanzi, a cominciare da Analisi in famiglia, la scrittrice ha analizzato l'evoluzione della condizione femminile al sud nel Novecento, raccontando la battaglia per l'affermazione della donna, che ha usato la scrittura come forma di espressione di sé, ricerca d'identità, occasione di riscatto. Marcone racconta, attraverso l'uso costante dell'autoanalisi, il dolore delle donne del sud, che spesso prende le forme patologiche e sfocia in segrete aspirazioni alla ribellione. La sua cifra stilistica è la linearità e la semplicità, che si traducono in un linguaggio

17. Marcone, Maria, Analisi in famiglia, Milano, Feltrinelli, 1977.

18. Campobasso, Antonio, Nero di Puglia, Milano, Feltrinelli, 1980.

19. Così Alfonso di Nola nella prefazione al volume.

20. Cfr. Pegorari, Daniele Maria, "La letteratura contemporanea in terra di Bari", in Catalano, Ettore (a cura di), La saggezza della letteratura, cit., p. 174. 
piano, immediato, caratterizzato spesso da un distacco ironico che le serve per traguardare anche le situazioni più tragiche.

Con gli anni Settanta ha inizio anche la stagione narrativa del barese Giorgio Saponaro, scrittore versatile e prolifico, apprezzato anche a livello nazionale grazie alla pubblicazione dei suoi racconti nella raccolta Racconti italiani del Novecento $^{21}$. Autore anche di una raccolta di poesie, alterna alla narrativa la saggistica di costume, come Il grillo parlante $e^{22}$, una "specie di summa e di distillato del suo vissuto di intellettuale e di uomo di mondo"23 o il romanzo Ilpellicano ${ }^{24}$, anch'esso specchio dei baresi in particolare e degli italiani in genere, nei loro vizi e nelle loro virtù quotidiane. La linea del realismo intimistico e della narrativa esistenziale $^{25}$ di questo romanzo prosegue con La trasgressione $e^{26}$, in cui rappresenta con ironia vizi e virtù di personaggi della piccola e la media borghesia meridionale. Più tardi, lo scrittore barese racconta con Il ragazzo di Tirana ${ }^{27}$ la vita di un clandestino albanese in Puglia, una storia di iniziazione e formazione, mentre in seguito torna a parlare della sua città con Il romanzo di Bari ${ }^{28}$.

Altro esempio di scrittore legato alla "baresità" è costituto da Tommaso Dell'Era di cui ricordiamo I cari baresi ${ }^{29}$. Vi racconta, anche lui con sottile ironia, i personaggi, le abitudini e la storia della città; è un libello in cui l'autore "castigat ridendo mores" dei suoi concittadini e attraverso loro mette in luce i costumi degenerati della borghesia nazionale. In questo pamphlet l'autore mostra una profonda sensibilità antropologica. In seguito, scrive I cavalieri di san Nicola $a^{30}:$ i "cavalieri" sono i sessantadue marinai che commisero il "sacro furto", il trafugamento delle reliquie di San Nicola. L'amore per la tradizione della città si ricongiunge alla storia di un viaggio che può ben essere considerato il mito della sua origine. L'opera deve la sua coralità proprio al carattere "collettivo" di questi cavalieri, che emblematicamente riassume quello dell'intera popolazione barese. $\mathrm{Si}$ possono ritenere quindi quelle di Dell'Era libere "spregiudicate analisi

21. Siciliano, Enzo, Racconti italiani del Novecento, Milano, collana "I Meridiani”, Mondadori, 2001.

22. Saponaro, Giorgio, Il grillo parlante, Bari, Adda, 1998.

23. Custodero, Gianni, Puglia letteraria nel Novecento. Poeti e prosatori, Ravenna, Longo 1982, pp. 138-139.

24. Saponaro, Giorgio, Il pellicano, Bari, Adda, 1981.

25. Cfr. Dell'Aquila, Michele, Parnaso di Puglia, Bari, Adda, 1983, p. 168.

26. Saponaro, Giorgio, La trasgressione, Bari, Adda, 1983.

27. ID., Il ragazzo di Tirana, Firenze, Giunti, 1996.

28. ID., Il romanzo di Bari, Bari, Adda, 2005.

29. Dell'Era, Tommaso, I cari baresi, Fasano, Schena, 1971.

30. ID., I cavalieri di san Nicola, Fasano, Schena, 1992. 
antropologiche condotte con penna lieve e felice, con uno stile costantemente tenuto a un livello colto, ancorché cordiale e moderno" ${ }^{\text {31 }}$.

Alla stessa école barisienne appartiene Vito Maurogiovanni, considerato da molti la "memoria storica" della città di Bari e il "cantore della sua gente". Sindacalista, giornalista, sceneggiatore radiofonico, scrittore e commediografo, ha scritto una trentina di libri tra cui ricordiamo due opere: Eravamo tutti Balilla ${ }^{32}$, in cui riattraversa l'esperienza degli anni del fascismo a Bari attraverso gli occhi di un ragazzo, e Nel tempo del silenzio e dei camini ${ }^{33}$, appunti e impressioni sulle vecchie masserie pugliesi rivisitate con attenzione commossa e attenta alla sua terra. Ha scritto anche trentadue commedie e un centinaio di radio-drammi in vernacolo e in lingua in cui il contrasto tra ricchezza e povertà si trasfigura nelle immagini degli splendori delle suppellettili d'oro o degli allestimenti teatrali degli spettacoli del Petruzzelli. Maurogiovanni pubblica anche un'antologia, La città e i giorni. Storie, sogni, ricordi $i^{34}$, che raccoglie, attraverso una selezione significativa, brani tratti dalle sue opere che ci consegnano il ritratto di una città e insieme del suo autore.

Al Mediterraneo come culla di una civiltà "meridiana”, luogo d'intersezione fra Oriente e Occidente e perciò irriducibile a una perimetrazione geo-culturale europea, africana o asiatica, guarda il poeta e romanziere Giuseppe Goffredo, che però preferisce rimanere estraneo a ogni sodalizio letterario, investendo maggiormente nel contatto con l'ineludibile teorizzazione sociologica e antropologica di Franco Cassano, com'è evidente nel suo volume Cadmos cerca Europa. Il Sud fra il Mediterraneo e l'Europa $a^{35}$, nel quale Goffredo ripensa al Sud Italia come officina permanente di una cultura di pace, contrapposta a quella militaristica di frontiera armata. In questo contesto s'inserisce l'attività di organizzatore culturale di Goffredo, all'interno del Laboratorio Progetto Poiesis, della cui sezione operativa è responsabile scientifico, e della rivista di letteratura e arte mediterranee Da Qui, da lui stesso diretta.

31. Guaragnella, Pasquale, Tommaso Dell'Era scrittore barese, in Strazzeri, Cosimo (a cura di), Un provinciale d'Europa, Bari, Progedit, 2007, p. 2.

32. Maurogiovanni, Vito, Eravamo tutti Balilla, Bari, Adda, 1970.

33. ID., Nel tempo del silenzio e dei camini, Bari, Levante, 1983.

34. ID., La città e i giorni. Storie, sogni, ricordi, Bari, Progedit, 2007.

35. Goffredo, Giuseppe, Cadmos cerca Europa. Il Sud fra il Mediterraneo e l'Europa, Torino, Bollati Boringhieri, 2000 (pubblicato anche ad Algeri in lingua francese). 
Rilevante è il ruolo occupato da Beppe Lopez che si è distinto sia come scrittore fin dal suo romanzo d'esordio ${ }^{36}$, sia come giornalista. Ma è soprattutto attraverso il lavoro di scrittore che Lopez mostra il suo legame con la terra natia. Lopez traccia il profilo di una città che fatica a diventare metropoli e vive nel disagio delle sue contraddizioni, ci consegna di Bari l'immagine di una città levantina che, a cavallo del secondo conflitto mondiale, vive una drammatica fase di crescita di cui è testimonianza la difficile condizione del sottoproletariato urbano degli anni Trenta e Quaranta, costretto all'arte della sopravvivenza. Di tale condizione è metafora la protagonista del romanzo, una ragazza di una bellezza provocante e selvaggia, figura della speranza e di un futuro negati (è una figlia non voluta, orfana, vive ai margini della città, vittima di violenze), a cui non resta che l'ostinazione per affermare la propria voglia di essere, quella che le merita proprio l'appellativo di "capatosta". Strumento straordinario per tradurre questa condizione sociale e individuale è il linguaggio che Lopez inventa, una lingua che rimanda a suoni e accenti di cui si sono smarrite le origini.

Francesco Dezio esordisce con i racconti pubblicati nell'antologia Sporco al sole, racconti del Sud estremo ${ }^{37}$, ma la sua fama è legata al suo primo romanzo, Nicola Rubino è entrato in fabbrica ${ }^{38}$. Collabora con vari quotidiani su cui spesso pubblica dei racconti come Popolo delle formiche ${ }^{39}$ e Sei troppo vecchio... hai 33 anni $^{40}$. Quest'ultimo è un racconto in prima persona di un trentatreenne che si ritrova disoccupato in un mercato del lavoro in cui ormai è considerato un "vecchio" ed è costretto a dei periodi di prova interminabili e sottopagati. Anche il primo racconto racconta della difficoltà di mantenere un lavoro nell'entroterra barese: il fratello della voce narrante viene licenziato e da questo evento ha origine una riflessione sul degrado economico della regione, sul modo che le aziende del settore hanno di affrontare la concorrenza cinese risparmiando su personale e materie prime nonostante i sovvenzionamenti statali "a pioggia". Nicola Rubino è entrato in fabbrica è considerato il primo esempio italiano di letteratura post industriale $e^{41}$, in bilico com'è tra la letteratura industriale degli anni Settanta e le

36. Lopez, Beppe, Capatosta, Milano, Mondadori, 2000. Con questo romanzo lo scrittore barese ha vinto il premio Bari contribuendo così a dare grande visibilità a questo evento letterario.

37. Trecca, Michele (a cura di), Sporco al sole, racconti del Sud estremo, Besa, Nardò, 1998.

38. Dezio, Francesco, Nicola Rubino è entrato in fabbrica, Milano, Feltrinelli, 2004.

39. ID., Popolo delle formiche, in Unità, 2004.

40. ID., Sei troppo vecchio... hai 33 anni, in Liberazione, 2014.

41. La dismissione, di Ermanno Rea (Milano, Feltrinelli 2002), viene spesso indicato come discrimine tra industriale e non. Si veda Chirumbolo, Paolo, Letteratura e lavoro, Soveria, Rubbettino, 2013. 
"scritture precarie" contemporanee. Dezio racconta appunto la storia di un operaio pugliese di trent'anni cui viene proposto un contratto di formazione da una multinazionale leader nel settore della produzione di motori diesel. Nicola, considerato una "testa calda", ricorda i suoi sei mesi di prova: un periodo infernale in cui "la fabbrica si prende tutto" (nulla di quanto raccontato nel romanzo avviene al di fuori dell'azienda), in cui a ritmi di lavoro disumani corrispondono continue vessazioni e minacce di licenziamento da parte dei capi e l'indifferenza dei colleghi. Scanditi dai ritmi ossessivi delle macchine della catena di montaggio e dal mobbing, i sei mesi si trasformano in un incubo. Così, quando il contratto a tempo indeterminato non arriva, per il protagonista è una liberazione. Per questo si è parlato di un anti-romanzo di formazione o di un romanzo di non-formazione umana e professionale. Dezio rielabora linguisticamente questo materiale biografico raccontandolo "con rabbia" attraverso un linguaggio che ha la forza polemica di un instant book, l'esattezza di un trattato di globalizzazione applicata, e insieme l'effetto trascinante della musica dei giovani d'oggi ${ }^{42}$.

Altro importante romanzo di questa nuova generazione di scrittori baresi è Occidente perprincipianti ${ }^{43}$ di Nicola Lagioia. Attraverso la vicenda del protagonista del suo romanzo, un intellettuale "precario" che si muove tra il mondo dei media, della politica e dell'accademia, constatando che l'oggi non può offrire che una condizione di assoluta solitudine, lo scrittore racconta questo mondo assolutamente negativo attraverso gli strumenti della comunicazione che gli sono propri. Temi e strutture della sua narrativa sono rappresentativi delle questioni fondamentali con cui la letteratura contemporanea è destinata a confrontarsi: dall'influenza della società del consumo e dello spettacolo al rapporto tra tecnologia e arte, dalla crisi del paradigma umanistico al recupero di centralità della figura dell'intellettuale ${ }^{44}$. Proprio per scacciare il terrore della morte, secondo Lagioia, l'uomo di oggi si affida alla figura-guida di santa Claus che esorcizza la paura di morire attraverso la distribuzione di regali. In Babbo Natale. Dove si racconta come la Coca-Cola ha plasmato il nostro immaginario ${ }^{45}$, Lagioia racconta del rapporto di interdipendenza e di affinità tra la figura di santa Claus (da elemento della cristianità

42. Cfr. Beretta, Alessandro, "Questa fabbrica è un inferno", in Corriere della sera, 10 ottobre 2004 e TreccA, Michele, "Tremate, l'operaio è tornato un po' incazzato", in La Gazzetta del Mezzogiorno, 21 settembre 2004.

43. Lagioia, Nicola, Occidente per principianti, Torino, Einaudi, 2004.

44. Cfr. Simonetti, Gianluigi, "Di fronte alla Medusa. Conversazione con Nicola Lagioia”, in Contemporanea, n. 4, 2006, pp. 215-221.

45. Lagioia, Nicola, Babbo Natale. Dove si racconta come la Coca-Cola ha plasmato il nostro immaginario, Roma, Fazi, 2005. 
a icona del consumismo) e la storia della Coca-Cola dimostrando come il sistema delle multinazionali sia capace di colonizzare il nostro immaginario. Le forme di mitopoiesi recenti quindi interessano Lagioia, come lui stesso dichiara; i personaggi dei suoi romanzi con le loro microstorie sono pedine nella scacchiera della macrostoria, divenendo così emblemi dell'uomo occidentale. Nel romanzo 2005 dopo Cristo ${ }^{46}$, lo scrittore denuncia la fossilizzazione del presente: nell'attuale era mediatica tutto è proteso verso il futuro e tutto è sotto l'influsso dei media, il presente nasce già passato. Questo non è necessariamente un danno, ma può essere invece occasione per l'artista di "riprendere la storia nel momento in cui accade" ${ }^{47}$. Tre sistemi per sbarazzarsi di Tolstoj (senza risparmiare se stessi) ${ }^{48}$ è infatti all'insegna della "schizofrenia tra passato e presente" (Tolstoj è trattato come un autore contemporaneo) e si configura come "radicale contestazione del concetto positivista di letteratura e storia ('La Storia non è maestra di niente')" ${ }^{49}$. Il tema dell'uomo nella società dei media e quello della scissione tra realtà reale e realtà virtuale (tra ciò che accade e la sua trasposizione nei media), costituiscono la spina dorsale dell'opera lagioiana. Questa tematica si ricongiunge a quella della Storia visto che, secondo Lagioia, la sua fine consiste nella sua derealizzazione e nella sua trasformazione in mito come conseguenza della mediatizzazione ${ }^{50}$.

"Un sud modaiolo che fa finta di vivere" Andrea Piva, Apocalisse da camera ${ }^{52}$. Si tratta di un sud irrimediabilmente contaminato dalla modernità che ne ha distrutto le radici e imbarbarito le tradizioni, iniettando il veleno di un consumismo malato e insieme straccione di cui Bari è emblema (ne sono testimonianze i fatiscenti bassi abitati dai pescatori trasformati nelle ricche residenze di avvocati, palazzinari e parrucchieri, la nuova "classe dirigente"). Qui ormai è tutto mortissimo, anche se tutto sembra sfrenatamente vivo e vitale. È l'apocalisse, appunto. Personifica questo mondo Ugo Cenci, un giovane rampollo di una famiglia della Bari "bene", con cui conserva un rapporto conflittuale; cresciuto male, è ossessionato dal sesso, beve, si

46. ID., 2005 dopo Cristo, Torino, Einaudi, 2005.

47. Casadei, Alberto, "Occidente per principianti' di Nicola Lagioia: da Bari a Roma all'Italia al mondo", in Italianistica, XXXVI, n. 1-2, 2007, p. 257.

48. Lagioia, Nicola, Tre sistemi per sbarazzarsi di Tolstoj (senza risparmiare se stessi), Roma, Minimum fax, 2001.

49. Pent, Sergio, "Oggi Tolstoj vive a Roma e si fa di Coca (Cola)", in La Stampa, 19 settembre 2001.

50. Ibid.

51. Così recita il titolo della recensione di LAPORTA, Filippo, "Un sud modaiolo che fa finta di vivere", in Corriere della sera, 19 novembre 2006.

52. PIVA, Andrea, Apocalisse da camera, Torino, Einaudi, 2006. 
droga e, in apparenza, è privo di qualsiasi morale, accetta compromessi e guarda con feroce disincanto, che lo fissa in un immobilismo senza riparo, la vita sua e quella degli altri. Assistente di Filosofia del diritto, utilizza il suo ruolo per ottenere favori sessuali dalle studentesse, ma quando qualcuno comincia a "parlare" tutto collassa nella sua vita, tutto precipita nella tragedia finale, che si sviluppa in un giorno. "Sulfureo, impietoso, classico - leggiamo nel risvolto di copertina - questo romanzo febbrile, scritto con suprema padronanza di stile" (anche il vernacolo subisce una profonda e sapiente elaborazione) ci parla di una "generazione viziata, pavida, inconsapevole e del tutto priva di ideali - che però nel momento della disfatta scopre non senza sorpresa di avere un carattere. E un cuore". Nel finale comico e tragico, insomma, grottesco, Ugo, ricoverato in ospedale, si consegna a quella dolce morte cui da sempre aspirava solo dopo aver riso a crepapelle.

Chiudiamo questa troppo rapida e lacunosa rassegna degli scrittori contemporanei della terra di Bari con il romanzo di Francesco Laudadio, Scrivano Ingannamorte ${ }^{53}$, "un libro indiscutibilmente necessario", come sostiene Andrea Camilleri nella prefazione. Le ragioni stanno nel suo carattere di militanza etico-civile che ci rimanda ai modelli forti della nostra cultura e ci consente, come afferma l'autore, di "ingannare la morte" attraverso la scrittura. Saggio storico, poema in prosa, racconto epico, attraverso le parole del narratore-protagonista che racconta di sé e dei suoi avi (di cui porta il soprannome e in cui si riconosce), il libro narra la storia di Rocco Sasso, segretario di una Camera del lavoro di un paese pugliese, che s'incontra con un giornalista che è andato a incontrarlo durante uno sciopero dei braccianti. Ed è la storia della lotta di classe nel sud che ha per protagonisti il seguace del cardinale Ruffo antifrancese e antigiacobino, il brigante in lotta contro i Savoia, il capopopolo delle leghe socialiste, il sindacalista negli anni difficili del secondo dopoguerra. È la storia di rivolte disperate e di spietate repressioni, "ferocia di bestia" per Laudadio. Dall'incipit del romanzo:

La notte passerà presto,vedrete, verrà giorno e non ce ne saremo accorti. È sempre così da noi, il giorno viene all'improvviso, un momento prima è tutto nero e pare che debba essere sempre notte e buio, poi di colpo è luce dappertutto.

53. Laudadio, Francesco, Scrivano Ingannamorte, Palermo, Sellerio, collana "La memoria", 2007. 
In guisa di conclusione, riportiamo i versi di una poesia di Anna Santoliquido, che raccontano di lei e dicono anche, in estrema sintesi, il carattere degli scrittori del sud:

Ho l'energia del Mezzogiorno $\backslash$ la testardaggine di chi ha partorito nei campi \e lottato con il padrone \gli stenti non mi hanno sconfitta \ho mischiato miele e fiele $\backslash$ attinti dalla terra $\backslash$ sono brigante e allodola $\backslash$ canto e maledico gli stolti \in me un oceano di fierezza \per la murgia le cattedrali le laure \le colline i castelli le foreste $\backslash$ rabbia e dolcezza mi contendono $\backslash$ sono ulivo e quercia $\backslash$ ginestra radicata alla costa ${ }^{54}$.

Giovanna ZACCARO

Università di Bari

54. Santoliquido, Anna, Stupor mundi, in La casa di pietra, Bucarest, Trocus, 2014, p. 134. 